\title{
GOLDIE EXTENDING MODULES AND GENERALIZATIONS OF QUASI-CONTINUOUS MODULES
}

\author{
By \\ Yosuke Kuratomi
}

\begin{abstract}
A module $M$ is said to be quasi-continuous if it is extending with the condition $\left(C_{3}\right)$ (cf. [7], [10]). In this paper, by using the notion of a $\mathscr{G}$-extending module which is defined by E. Akalan, G. F. Birkenmeier and A. Tercan [1], we introduce a generalization of quasi-continuous "a GQC(generalized quasicontinuous)-module" and investigate some properties of GQCmodules. Initially we give some properties of a relative ejectivity which is useful in analyzing the structure of $\mathscr{G}$-extending modules and GQC-modules (cf. [1]). And we apply them to the study of direct sums of GQC-modules. We also prove that any direct summand of a GQC-module with the finite internal exchange property is GQC. Moreover, we show that a module $M$ is $\mathscr{G}$-extending modules with $\left(C_{3}\right)$ if and only if it is GQC-module with the finite internal exchange property.
\end{abstract}

\section{Preliminaries}

Throughout this paper $R$ is a ring with identity and all modules considered are unitary right $R$-modules. A submodule $X$ of a module $M$ is said to be essential in $M$ or an essential submodule of $M$, if $X \cap Y \neq 0$ for any non-zero submodule $Y$ of $M$ and we write $X \subseteq_{e} M$ in this case. $X<_{\oplus} M$ means that $X$ is a direct summand of $M$. Let $M=A \oplus B$ and let $\varphi: A \rightarrow B$ be a homomorphism. Then $\langle A \stackrel{\varphi}{\rightarrow} B\rangle=\{a-\varphi(a) \mid a \in A\}$ is a submodule of $M$. Note that $M=A \oplus B=\langle A \stackrel{\varphi}{\rightarrow} B\rangle \oplus B$.

2010 Mathematics Subject Classification: Primary 16D10; Secondary 16D50.

Key words and phrases: Goldie-extending module, ejective module, finite internal exchange property. Received November 29, 2012.

Revised July 31, 2013. 
Let $\left\{M_{i} \mid i \in I\right\}$ be a family of modules. The direct sum decomposition $M=\bigoplus_{i \in I} M_{i}$ is said to be exchangeable if, for any direct summand $X$ of $M$, there exists $\overline{M_{i}} \subseteq M_{i}(i \in I)$ such that $M=X \oplus\left(\oplus_{i \in I} \overline{M_{i}}\right)$. A module $M$ is said to have the finite internal exchange property (FIEP) if, any finite direct sum decomposition $M=M_{1} \oplus \cdots \oplus M_{n}$ is exchangeable.

A module $M$ is said to be extending if, for any submodule $X$ of $M$, there exists a direct summand $A$ of $M$ such that $X$ is essential in $A$. A module $M$ is said to be $\mathscr{G}$-extending or Goldie extending if, for any submodule $X$ of $M$, there exist an essential submodule $X^{\prime}$ of $X$ and a direct summand $A$ of $M$ such that $X^{\prime}$ is essential in $A$. A module $M$ is said to be $\mathscr{G}^{+}$-extending if any direct summand of $M$ is $\mathscr{G}$-extending (cf. [1]). From [6], $\mathscr{G}$-extending modules with FIEP are $\mathscr{G}^{+}$-extending. Now we consider the following condition:

$\left(C_{3}\right)$ If $A$ and $B$ are direct summands of $M$ such that $A \cap B=0$, then $A \oplus B$ is a direct summand of $M$.

A module $M$ is said to be quasi-continuous if $M$ is extending with $\left(C_{3}\right)$ (cf. [7], [10]). We obtain that $M$ is a quasi-continuous module if and only if, for every submodules $X_{1}$ and $X_{2}$ of $M$ with $X_{1} \cap X_{2}=0$, there exists a decomposition $M=A_{1} \oplus A_{2}$ such that $X_{i} \subseteq A_{i}(i=1,2)$ (cf. [15, pp. 367-368]). Motivated by this result, we introduce a generalization of a quasi-continuous module as follows:

A module $M$ is said to be GQC (generalized quasi-continuous) if, for every submodules $X_{1}$ and $X_{2}$ with $X_{1} \cap X_{2}=0$, there exist an essential submodule $X_{i}^{\prime} \subseteq_{e} X_{i}$ and a decomposition $M=A_{1} \oplus A_{2}$ such that $X_{i}^{\prime}$ is a submodule of $A_{i}$ $(i=1,2)$. Note that any GQC-module is $\mathscr{G}$-extending (cf. Proposition 2.3).

Let $M_{\mathbf{Z}}=\mathbf{Z} / 2 \mathbf{Z} \oplus \mathbf{Z} / 8 \mathbf{Z}$. $\mathbf{Z} / 2 \mathbf{Z}$ and $\mathbf{Z} / 8 \mathbf{Z}$ are GQC-modules, but $M$ is not GQC (cf. Proposition 2.6). Hence a direct sum of GQC-modules need not be GQC. Moreover, it is unknown to the author whether or not the property GQC is inherited by direct summands.

In this paper, our main purpose is to show the following:

(I) Let $M_{1}$ and $M_{2}$ be GQC-modules with FIEP and put $M=M_{1} \oplus M_{2}$. Then $M$ is a GQC-module with FIEP if and only if $M_{i}$ is $M_{j}$-ejective $(i \neq j)$ and the decomposition $M=M_{1} \oplus M_{2}$ is exchangeable.

(II) If $M$ is a GQC-module with FIEP, then $A$ is GQC for any direct summand $A$ of $M$.

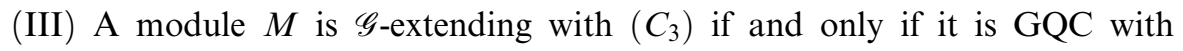
FIEP.

For undefined terminologies, the reader is referred to [2], [3], [7] and [15]. 
Many of the following lemmas can be found in the cited literature, but we list them here for easy reference.

Lemma 1.1. Let $M$ be a module with a decomposition $M=A \oplus B$ and let $X$ be a submodule of $M$. If $A \cap X \subseteq_{e} A$, then $X \supseteq_{e}(A \cap X) \oplus(B \cap X)$.

Proof. By [11, Lemma 2.2].

Lemma 1.2. Let $M=A \oplus B, C \subseteq A$ and let $f: C \rightarrow B$ be a homomorphism. If $X \subseteq_{e}\langle C \stackrel{f}{\rightarrow} B\rangle$, then there exists $C^{\prime} \subseteq_{e} C$ such that $X=\left\langle C^{\prime} \stackrel{\left.f\right|_{C^{\prime}}}{\longrightarrow} B\right\rangle$.

Proof. Evident.

Lemma 1.3. If $M=A \oplus B=X \oplus Y \oplus B$, then there exists a homomorphism $\alpha: X \oplus Y \rightarrow B$ such that $A=\langle X \oplus Y \stackrel{\alpha}{\rightarrow} B\rangle=\left\langle X \stackrel{\left.\alpha\right|_{X}}{\rightarrow} B\right\rangle \oplus\left\langle Y \stackrel{\left.\alpha\right|_{Y}}{\rightarrow} B\right\rangle$.

Proof. Let $p_{1}: M=X \oplus Y \oplus B \rightarrow X \oplus Y$ and $p_{2}: M=X \oplus Y \oplus B \rightarrow B$ be the projections. Define $\alpha: p_{1}(A) \rightarrow p_{2}(A)$ by $\alpha\left(p_{1}(a)\right)=p_{2}(a)$, where $a \in A$. Then $A=\langle X \oplus Y \stackrel{\alpha}{\rightarrow} B\rangle=\left\langle X \stackrel{\left.\alpha\right|_{X}}{\rightarrow} B\right\rangle \oplus\left\langle Y \stackrel{\left.\alpha\right|_{Y}}{\rightarrow} B\right\rangle$.

Lemma 1.4 (cf. [4], [9, Proposition 2.5]). Let $M=A \oplus B$. Then $M$ has FIEP if and only if $A$ and $B$ have FIEP and the decomposition $M=A \oplus B$ is exchangeable.

Let $A$ and $B$ be modules. $A$ is said to be essentially $B$-injective if, for any submodule $X$ of $B$ and any homomorphism $f: X \rightarrow A$ with $\operatorname{ker} f \subseteq_{e} X$, there exists a homomorphism $g: B \rightarrow A$ such that $\left.g\right|_{X}=f$.

Lemma 1.5. Let $A$ and $B$ be modules. If $A$ is essentially B-injective, then $A^{\prime}$ is essentially $B^{\prime}$-injective for any $A^{\prime}<_{\oplus} A$ and any $B^{\prime} \subseteq B$.

Proof. By [3, 2.15].

\section{Ejective Modules and GQC-modules}

Firstly, we recall a generalization of relative injectivity which is introduced by E. Akalan, G. F. Birkenmeier and A. Tercan [1]. 
Definition. Let $A$ and $B$ be modules. $A$ is said to be $B$-ejective if, for any submodule $X$ of $B$ and any homomorphism $f: X \rightarrow A$, there exist an essnetial submodule $X^{\prime}$ of $X$ and a homomorphism $g: B \rightarrow A$ such that $\left.g\right|_{X^{\prime}}=\left.f\right|_{X^{\prime}}$.

Now we consider some properties of relative ejectivities.

Proposition 2.1. Let $A, B, A_{i}$ and $B_{i}(i=1,2)$ be modules.

(1) If $A$ is B-ejective, then $A^{\prime}$ is $B^{\prime}$-ejective for any $A^{\prime}<_{\oplus} A$ and $B^{\prime} \subseteq B$.

(2) If $A$ is $B_{i}$-ejective $(i=1,2)$, then $A$ is $B_{1} \oplus B_{2}$-ejective.

(3) If $A_{i}$ is B-ejective $(i=1,2)$, then $A_{1} \oplus A_{2}$ is B-ejective.

ProOF. (1) is clear.

(2) Put $B=B_{1} \oplus B_{2}$, let $X$ be a submodule of $B$ and let $f: X \rightarrow A$ be a homomorphism. Let $Y$ be a complement of $X$ in $B$. Define $f^{*}: X \oplus Y \rightarrow A$ by $f^{*}(x+y)=f(x)$, where $x \in X$ and $y \in Y$. By $X \oplus Y \subseteq_{e} B,(X \oplus Y) \cap B_{i} \subseteq_{e} B_{i}$ $(i=1,2)$. Since $A$ is $B_{i}$-ejective $(i=1,2)$, there exist an essential submodule $B_{i}^{\prime}$ of $(X \oplus Y) \cap B_{i}$ and a homomorphism $g_{i}: B_{i} \rightarrow A$ such that $\left.g_{i}\right|_{B_{i}^{\prime}}=\left.f^{*}\right|_{B_{i}^{\prime}}(i=1,2)$. By $B_{1}^{\prime} \oplus B_{2}^{\prime} \subseteq_{e} X \oplus Y$, we see $\left(B_{1}^{\prime} \oplus B_{2}^{\prime}\right) \cap X \subseteq_{e} X$. Put $g=g_{1}+g_{2}: B \rightarrow A$. Let $x=b_{1}^{\prime}+b_{2}^{\prime} \in\left(B_{1}^{\prime} \oplus B_{2}^{\prime}\right) \cap X$, where $b_{i}^{\prime} \in B_{i}^{\prime}(i=1,2)$. Then

$$
f(x)=f^{*}(x)=f^{*}\left(b_{1}^{\prime}\right)+f^{*}\left(b_{2}^{\prime}\right)=g_{1}\left(b_{1}^{\prime}\right)+g_{2}\left(b_{2}^{\prime}\right)=g\left(b_{1}^{\prime}+b_{2}^{\prime}\right)=g(x) .
$$

Thus $A$ is $B$-ejective.

(3) Put $A=A_{1} \oplus A_{2}$, let $X$ be a submodule of $B$ and let $f: X \rightarrow A$ be a homomorphism. Let $p_{i}: A \rightarrow A_{i}$ be the projection $(i=1,2)$. Since $A_{i}$ is $B$-ejective, for $p_{i} f: X \rightarrow A_{i}$, there exist an essential submodule $X_{i}$ of $X$ and a homomorphism $g_{i}: B \rightarrow A_{i}$ such that $\left.g_{i}\right|_{X_{i}}=\left.p_{i} f\right|_{X_{i}}(i=1,2)$. By $X_{i} \subseteq_{e} X$ $(i=1,2), X_{1} \cap X_{2} \subseteq_{e} X$. Put $g=g_{1}+g_{2}: B \rightarrow A$. Then, for any $x \in X_{1} \cap X_{2}$,

$$
f(x)=p_{1} f(x)+p_{2} f(x)=g_{1}(x)+g_{2}(x)=g(x) .
$$

Thus $A$ is $B$-ejective.

Let $A$ and $B$ be modules. $A$ is said to be mono-B-injective if, for any submodule $X$ of $B$ and any monomorphism $f: X \rightarrow A$, there exists a homomorphim $g: B \rightarrow A$ such that $\left.g\right|_{X}=f$ (cf. [5]). The following is a connection between relative mono-injectivities and relative ejectivities.

Proposition 2.2. Let $A$ be a module and let $B$ be a $\mathscr{G}$-extending module. If $A$ is mono-B-injective, then $A$ is B-ejective. 
Proof. Let $X$ be a submodule of $B$ and let $f: X \rightarrow A$ be a homomorphism. As $B$ is $\mathscr{G}$-extending, there exist an essential submodule $K$ of $\operatorname{ker} f$ and a decomposition $B=B_{1} \oplus B_{2}$ such that $K \subseteq_{e} B_{1}$. By Lemma 1.1, $X \supseteq_{e} K \oplus\left(B_{2} \cap X\right)$. Then $\left.f\right|_{B_{2} \cap X}$ is a monomorphism. Since $A$ is mono- $B_{2}$-injective, there exists a homomorphism $g: B_{2} \rightarrow A$ with $\left.g\right|_{B_{2} \cap X}=\left.f\right|_{B_{2} \cap X}$. Define $h: B \rightarrow A$ by $h\left(b_{1}+b_{2}\right)=g\left(b_{2}\right)$, where $b_{i} \in B_{i} \quad(i=1,2)$. Let $k+b_{2} \in K \oplus\left(B_{2} \cap X\right)$, where $k \in K$ and $b_{2} \in B_{2} \cap X$. Then $h\left(k+b_{2}\right)=g\left(b_{2}\right)=f\left(b_{2}\right)=f\left(k+b_{2}\right)$. Hence $A$ is $B$-ejective.

Now we show that any GQC-module is $\mathscr{G}$-extending.

Proposition 2.3. If $M$ is a GQC-module, then it is $\mathscr{G}$-extending.

Proof. Let $M$ be a GQC-module and let $X$ be a submodule of $M$. Let $Y$ be a complement of $X$ in $M$. By $X \cap Y=0$, there exists essential submodules $X^{\prime}$ of $X$ and $Y^{\prime}$ of $Y$ and a decomposition $M=A \oplus B$ such that $X^{\prime} \subseteq A$ and $Y^{\prime} \subseteq B$. Then $X^{\prime}=\left(X^{\prime} \oplus Y^{\prime}\right) \cap A \subseteq_{e} M \cap A=A$. Thus $M$ is $\mathscr{G}$-extending.

Proposition 2.4. If $M$ is a $\mathscr{G}$-extending module with $\left(C_{3}\right)$, then it is $G Q C$.

Proof. Obvious.

By [1, Example 3.20], $M_{\mathbf{Z}}=\mathbf{Q} \oplus \mathbf{Z} / p \mathbf{Z}$ is a $\mathscr{G}$-extending module with $\left(C_{3}\right)$ but not quasi-continuous, where $p$ is a prime number.

Now we give a characterlization for any direct summand of a GQC-module to be GQC.

Proposition 2.5. If $M$ is a GQC-module with FIEP, then $A$ is GQC for any direct summand $A$ of $M$.

Proof. Let $A$ be a direct summand of $M$ and let $X$ and $Y$ be submodules of $A$ with $X \cap Y=0$. As $M$ is GQC, there exist essential submodules $X^{\prime}$ of $X$ and $Y^{\prime}$ of $Y$ and a decomposition $M=M_{1} \oplus M_{2}$ such that $X^{\prime} \subseteq M_{1}$ and $Y^{\prime} \subseteq M_{2}$. Since $M$ satisfies FIEP, there exists a direct summand $M_{i}^{\prime}$ of $M_{i}$ $(i=1,2)$ such that $M=A \oplus M_{1}^{\prime} \oplus M_{2}^{\prime}$. Put $M_{i}=M_{i}^{\prime} \oplus M_{i}^{\prime \prime} \quad(i=1,2)$. By Lemma 1.3, there exists a homomorphism $\alpha: M_{1}^{\prime \prime} \oplus M_{2}^{\prime \prime} \rightarrow M_{1}^{\prime} \oplus M_{2}^{\prime}$ such 
that $\quad A=\left\langle M_{\alpha}^{\prime \prime} \stackrel{\left.\alpha\right|_{M_{1}^{\prime \prime}}}{\longrightarrow} M_{1}^{\prime} \oplus M_{2}^{\prime}\right\rangle \oplus\left\langle M_{2}^{\prime \prime} \stackrel{\alpha_{M_{2}^{\prime \prime}}}{\longrightarrow} M_{1}^{\prime} \oplus M_{2}^{\prime}\right\rangle$. By ${ }_{\left.\alpha\right|_{M^{\prime \prime}}} \quad X^{\prime} \subseteq A \cap M_{1}, \quad X^{\prime} \subseteq$ $\left\langle M_{1}^{\prime \prime} \stackrel{\alpha{ }_{M_{1}^{\prime \prime}}}{\longrightarrow} M_{1}^{\prime} \oplus M_{2}^{\prime}\right\rangle$. Similarly, we obtain $Y^{\prime} \subseteq\left\langle M_{2}^{\prime \prime} \stackrel{\left.{ }^{\prime \prime}\right|_{M_{2}^{\prime \prime}}}{\longrightarrow} M_{1}^{\prime} \oplus M_{2}^{\prime}\right\rangle$. Thus $A$ is GQC.

Let $M$ be a finitely generated torsion-free abelian group with rank $\geq 2$. Then $M$ is $(\mathscr{G}$-)extending but not satisfy FIEP (cf. [3, p. 56]). For $\mathscr{G}$-extending modules with FIEP, we can give a characterization of GQC-module in a term of a relative ejectivity as follows:

Proposition 2.6. Let $M$ be a G-extending module with FIEP. Then $M$ is $G Q C$ if and only if $A$ is $B$-ejective for any decomposition $M=A \oplus B$.

Proof. $(\Rightarrow)$ Let $M=A \oplus B$, let $X$ be a submodule of $B$ and let $f: X \rightarrow A$ be a homomorphism. As $B$ is $\mathscr{G}$-extending, there exist an essential submodule $X^{\prime}$ of $X$ and a decomposition $B=B_{1} \oplus B_{2}$ such that $X^{\prime} \subseteq_{e} B_{1}$. By Proposition 2.5, $A \oplus B_{1}$ is GQC and so we may assume that $X \subseteq_{e} B$.

By $\langle X \stackrel{f}{\rightarrow} A\rangle \cap A=0$, there exist essential submodules $T$ of $\langle X \stackrel{f}{\rightarrow} A\rangle$ and $L$ of $A$ and a decomposition $M=M_{1} \oplus M_{2}$ such that $T \subseteq M_{1}$ and $L \subseteq M_{2}$. By $\langle X \stackrel{f}{\rightarrow} A\rangle \oplus A=X \oplus A \subseteq_{e} M$, we see $T \subseteq_{e} M_{1}$ and $L \subseteq_{e} M_{2}$. Since $M$ satisfies FIEP, there exists $M_{i}^{\prime} \subseteq M_{i}(i=1,2)$ such that $M=A \oplus M_{1}^{\prime} \oplus M_{2}^{\prime}$. By $L \subseteq_{e} M_{2} \cap A$, we get $M_{2}^{\prime}=0$ and so $M=A \oplus M_{1}^{\prime}$. By $A \oplus M_{1} \subseteq_{e} M$, we obtain $M=A \oplus M_{1}$. By Lemma 1.3, there exists a homomorphism $g: B \rightarrow A$ such that $M_{1}=\langle B \stackrel{g}{\rightarrow} A\rangle$.

As $T \subseteq_{e}\langle X \stackrel{f}{\rightarrow} A\rangle$, by Lemma 1.2, there exists an essential submodule $X^{\prime}$ of $X$ such that $T=\left\langle X^{\prime} \stackrel{\left.f\right|_{X^{\prime}}}{\longrightarrow} A\right\rangle$. Thus $\left\langle X^{\prime} \stackrel{\left.f\right|_{X^{\prime}}}{\longrightarrow} A\right\rangle=T \subseteq_{e} M_{1}=\langle B \stackrel{g}{\rightarrow} A\rangle$. Then, for any $x^{\prime} \in X^{\prime}$, there exists $b \in B$ such that $x^{\prime}-f\left(x^{\prime}\right)=b-g(b)$. By $x^{\prime}=b$ and $f\left(x^{\prime}\right)=g(b)$, we obtain $g\left(x^{\prime}\right)=f\left(x^{\prime}\right)$. Therefore $A$ is $B$-ejective.

$(\Leftarrow)$ Let $X$ and $Y$ be submodules of $M$ with $X \cap Y=0$. As $M$ is $\mathscr{G}$ extending, there exist an essential submodule $X^{\prime}$ of $X$ and a decomposition $M=$ $A \oplus B$ such that $X^{\prime} \subseteq_{e} A$. Let $p_{A}: M=A \oplus B \rightarrow A$ and $p_{B}: M=A \oplus B \rightarrow B$ be the projections. By $Y \cap A=0$, we can define a homomorphism $f: p_{B}(Y) \rightarrow$ $p_{A}(Y)$ by $f\left(p_{B}(y)\right)=p_{A}(y)$, where $y \in Y$. Since $A$ is $B$-ejective, there exist an essential submodule $B^{\prime}$ of $p_{B}(Y)$ and a homomorphism $g: B \rightarrow A$ such that $\left.g\right|_{B^{\prime}}=\left.f\right|_{B^{\prime}}$. Then we see

$$
\left\langle B^{\prime} \stackrel{\left.f\right|_{B^{\prime}}}{\longrightarrow} A\right\rangle \subseteq \subseteq_{e}\left\langle p_{B}(Y) \stackrel{f}{\rightarrow} p_{A}(Y)\right\rangle=Y \quad \text { and } \quad\left\langle B^{\prime} \stackrel{\left.f\right|_{B^{\prime}}}{\longrightarrow} A\right\rangle \subseteq\langle B \stackrel{g}{\rightarrow} A\rangle .
$$

Thus $M$ is GQC. 
Let $M_{\mathbf{Z}}=\mathbf{Z} / 2 \mathbf{Z} \oplus \mathbf{Z} / 8 \mathbf{Z}$. From [1, Example 3.4] or [6], we see that $M$ is $\mathscr{G}_{-}$ extending with FIEP. However, by Proposition 2.6, $M$ is not GQC since $\mathbf{Z} / 2 \mathbf{Z}$ is not $\mathbf{Z} / 8 \mathbf{Z}$-ejective. Next we show a characterization for a GQC-module to be quasi-continuous.

Proposition 2.7. Let $M$ be a $\mathscr{G}$-extending module. Assume that $A$ is essentially B-injective for any decomposition $M=A \oplus B$. Then

(1) $M$ is extending.

(2) $M$ satisfies $\left(C_{3}\right)$ if and only if it is GQC.

Proof. (1) By [6, Proposition 2.1].

$(2)(\Rightarrow)$ By Proposition 2.4.

$(\Leftarrow)$ Let $X$ and $Y$ be direct summands of $M$ with $X \cap Y=0$. Since $M$ is GQC, there exist essential submodules $X^{\prime} \subseteq_{e} X$ and $Y^{\prime} \subseteq_{e} Y$ and a decomposition $M=A \oplus B$ such that $X^{\prime} \subseteq A$ and $Y^{\prime} \subseteq_{e} B$. Let $p_{A}: M=A \oplus B \rightarrow A$ and $p_{B}: M=A \oplus B \rightarrow B$ be the projections. By $Y \cap A=0$ and $Y^{\prime} \subseteq_{e} Y$, the canonical map $f: p_{B}(Y) \rightarrow p_{A}(Y)$ is a homomorphism with $\operatorname{ker} f \subseteq_{e} p_{B}(Y)$. Since $A$ is essentially $B$-injective, there exists a homomorphism $f^{*}: B \rightarrow A$ such that $\left.f^{*}\right|_{p_{B}(Y)}=f$ and then

$$
M=\left\langle B \stackrel{f^{*}}{\rightarrow} A\right\rangle \oplus A \quad \text { and } \quad Y=\left\langle p_{B}(Y) \stackrel{f}{\rightarrow} p_{A}(Y)\right\rangle \subseteq_{e}\left\langle B \stackrel{f^{*}}{\rightarrow} A\right\rangle .
$$

Hence $M=Y \oplus A$. By (1), there exists a decomposition $A=A^{\prime} \oplus A^{\prime \prime}$ such that $X^{\prime} \subseteq_{e} A^{\prime}$. By the same argument above, there exists a homomorphism $g^{*}: A^{\prime} \rightarrow Y \oplus A^{\prime \prime}$ such that $M=\left\langle A^{\prime} \stackrel{g^{*}}{\rightarrow} Y \oplus A^{\prime \prime}\right\rangle \oplus Y \oplus A^{\prime \prime}$ and $X \subseteq_{e}\left\langle A^{\prime} \stackrel{g^{*}}{\rightarrow}\right.$ $\left.Y \oplus A^{\prime \prime}\right\rangle$. Thus $M=X \oplus Y \oplus A^{\prime \prime}$.

Corollary 2.8. Let $M$ be a module. Then $M$ is quasi-continuous if and only if $M$ is GQC and $A$ is essentially B-injective for any decomposition $M=A \oplus B$.

Proof. By Proposition 2.7 and [7, Proposition 2.10].

Now we give a necessary and sufficient condition for a direct sum of GQC-modules with FIEP to be GQC with FIEP. First, we show the following lemma which is due to E. Akalan, G. F. Birkenmeier and A. Tercan [1, Theorem 3.1]. 
Lemma 2.9 ([1, Theorem 3.1]). Let $A$ and $B$ be $\mathscr{G}$-extending modules and put $M=A \oplus B$. If $A$ is $B$-ejective, then $M$ is $\mathscr{G}$-extending. In general, the converse is not true.

The following is an immediate consequence of Proposition 2.2 and Lemma 2.9 .

Corollary 2.10. Let $A$ and $B$ be $\mathscr{G}$-extending modules and put $M=A \oplus B$. If $A$ is mono-B-injective, then $M$ is $\mathscr{G}$-extending.

By Proposition 2.6 and Lemma 2.9, we obtain the following result.

THEOREM 2.11. Let $M_{1}$ and $M_{2}$ be GQC-modules with FIEP and put $M=M_{1} \oplus M_{2}$. Then $M$ is a GQC-module with FIEP if and only if $M_{i}$ is $M_{j}$ ejective $(i \neq j)$ and the decomposition $M=M_{1} \oplus M_{2}$ is exchangeable.

Proof. $\quad(\Rightarrow)$ By Proposition 2.6.

$(\Leftarrow)$ By Lemmas 1.4 and $2.9, M$ is $\mathscr{G}$-extending with FIEP. By Proposition 2.6, we may prove that $A$ is $B$-ejective for any decomposition $M=A \oplus B$. Let $M=A \oplus B$. Since the decomposition $M=M_{1} \oplus M_{2}$ is exchangeable, there exists $M_{i}^{\prime} \subseteq M_{i}(i=1,2)$ such that $M=A \oplus M_{1}^{\prime} \oplus M_{2}^{\prime}$. Put $M_{i}=M_{i}^{\prime} \oplus M_{i}^{\prime \prime}(i=1,2)$. Then

$$
A \simeq M_{1}^{\prime \prime} \oplus M_{2}^{\prime \prime} \quad \text { and } \quad B \simeq M_{1}^{\prime} \oplus M_{2}^{\prime}
$$

As $M_{i}$ is GQC-module with FIEP, by Proposition 2.6, $M_{i}^{\prime \prime}$ is $M_{i}^{\prime}$-ejective $(i=1,2)$. By Proposition 2.1, we see that $M_{1}^{\prime \prime} \oplus M_{2}^{\prime \prime}$ is $M_{1}^{\prime} \oplus M_{2}^{\prime}$-ejective. Hence $A$ is $B$-ejective. Thus $M$ is a GQC-module with FIEP.

By results above, we can easily prove the following result which is well known:

COROLlary 2.12 ([7, Theorem 2.13]). Let $M_{1}$ and $M_{2}$ be quasi-continuous modules and put $M=M_{1} \oplus M_{2}$. Then $M$ is quasi-continuous if and only if $M_{i}$ is $M_{j}$-injective $(i \neq j)$. 
Proof. $(\Rightarrow)$ By Theorem 2.11 and [4, Proposition 1.4 and Theorem 2.1], $M_{i}$ is $M_{j}$-ejective and essentially $M_{j}$-injective $(i \neq j)$. Thus $M_{i}$ is $M_{j}$-injective $(i \neq j)$ by [6, Proposition 2.2].

$(\Leftarrow)$ By [4, Proposition 1.4, Theorems 2.1 and 2.15], we see that $M$ is extending with FIEP and $A$ is essentially $B$-injective for any decomposition $M=A \oplus B$. Thus, by Proposition 2.7 and Theorem 2.11, $M$ is quasi-continuous.

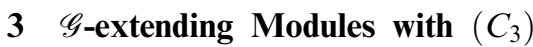

Firstly we recall the condition $\left(C_{11}\right)$ from [12], which can be considered as a generalization of $\mathscr{G}$-extending.

Definition (cf. [12]). Let $M$ be a module. $M$ is said to be a $\left(C_{11}\right)$-module if any submodule $X$ of $M$ has a complement which is a direct summand of M.

From [13, Example 4], there exists a $\left(C_{11}\right)$-module which has a direct summand that does not satisfy $\left(C_{11}\right)$. However, any direct summand of $\left(C_{11}\right)$-modules with $\left(C_{3}\right)$ satisfies $\left(C_{11}\right)$.

Proposition 3.1. Let $M$ be a $\left(C_{11}\right)$-module with $\left(C_{3}\right)$ and let $A$ be a direct summand of $M$. Then $A$ is a $\left(C_{11}\right)$-module with $\left(C_{3}\right)$.

Proof. Let $M$ be a $\left(C_{11}\right)$-module with $\left(C_{3}\right)$ and let $M=A \oplus B$. From [7, Proposition 2.7], we may show that $A$ satisfies $\left(C_{11}\right)$. Let $X$ be a submodule of $A$. Since $M$ satisfies $\left(C_{11}\right)$, there exists a direct summand $N$ of $M$ such that $(X \oplus B) \oplus N \subseteq_{e} M$. By $\left(C_{3}\right), B \oplus N$ is a direct summand of $M$. Put $M=$ $T \oplus N \oplus B$. By Lemma 1.3, there exists a homomorphism $\alpha: T \oplus N \rightarrow B$ such that $A=\left\langle T \stackrel{\left.\alpha\right|_{T}}{\rightarrow} B\right\rangle \oplus\left\langle N \stackrel{\left.\alpha\right|_{N}}{\rightarrow} B\right\rangle$. Put $A_{1}=\left\langle T \stackrel{\left.\alpha\right|_{T}}{\rightarrow} B\right\rangle$ and $A_{2}=(N \oplus B) \cap A$. Then we see $M=A_{1} \oplus N \oplus B$ and $A=A_{1} \oplus A_{2}$.

Now we prove that $X \oplus A_{2} \subseteq_{e} A$. Given $0 \neq a \in A$ and express $a$ in $A=A_{1} \oplus A_{2}$ as $a=a_{1}+a_{2}\left(a_{i} \in A_{i}\right)$. If $a_{1}=0$, then $0 \neq a=a_{2} \in A_{2}$. Let $a_{1} \neq 0$. By $X \oplus B \oplus N \subseteq_{e} M$, there exists $r \in R$ such that $0 \neq a_{1} r=x+b+n$ for some $x \in X, b \in B$ and $n \in N$. So $n+b=a_{1} r-x \in(N \oplus B) \cap A=A_{2}$. Thus $0 \neq a r=$ $a_{1} r+a_{2} r=x+\left(b+n+a_{2} r\right) \in X \oplus A_{2}$. Hence $X \oplus A_{2} \subseteq_{e} A$.

Therefore $A_{2}$ is a complement of $X$ in $A$. 
Now we consider the following conditions for a module $M$ (cf. [1], [14]):

(*) For any decomposition $M=A \oplus B, A$ is $B$-ejective.

(SIP) For any direct summands $A$ and $B$ of $M, A \cap B$ is a direct summand.

Proposition 3.2. If $M$ is module with the conditions $(*)$ and $(S I P)$, then $M$ satisfies $\left(C_{3}\right)$.

Proof. Let $A$ and $B$ be direct summands of $M$ with $A \cap B=0$. Put $M=A \oplus C$. Let $p_{A}: M \rightarrow A$ and $p_{C}: M \rightarrow C$ be the projections. Define $f: p_{C}(B) \rightarrow p_{A}(B)$ by $f\left(p_{C}(b)\right)=p_{A}(b)$, where $b \in B$. Since $A$ is $C$-ejective, there exist an essntial submodule $C^{\prime}$ of $p_{C}(B)$ and a homomorphism $g: C \rightarrow A$ such that $\left.g\right|_{C^{\prime}}=\left.f\right|_{C^{\prime}}$. Then

$$
\left\langle C^{\prime} \stackrel{\left.f\right|_{C^{\prime}}}{\longrightarrow} A\right\rangle \subseteq\langle C \stackrel{g}{\rightarrow} A\rangle \text { and }\left\langle C^{\prime} \stackrel{\left.f\right|_{C^{\prime}}}{\longrightarrow} A\right\rangle \subseteq \subseteq_{e}\left\langle p_{C}(B) \stackrel{f}{\rightarrow} p_{A}(B)\right\rangle=B .
$$

So we see $\langle C \stackrel{g}{\rightarrow} A\rangle \cap B \subseteq_{e} B$. By $(S I P),\langle C \stackrel{g}{\rightarrow} A\rangle \cap B$ is a direct summand of $M$ and hence $\langle C \stackrel{g}{\rightarrow} A\rangle \cap B=B$. As $B \subseteq\langle C \stackrel{g}{\rightarrow} A\rangle$, there exists a direct summand $T$ of $M$ such that $\langle C \stackrel{g}{\rightarrow} A\rangle=B \oplus T$. Thus $M=A \oplus B \oplus T$.

Next we show that any $\left(C_{11}\right)$-module with $(*)$ is $\mathscr{G}$-extending.

Proposition 3.3. Let $M$ be a module with (*). Then $M$ is $\mathscr{G}$-extending if and only if $M$ satisfies $\left(C_{11}\right)$.

Proof. $(\Rightarrow)$ is clear.

$(\Leftarrow)$ Let $X$ be a submodule of $M$. Then there exists a direct summand $A$ of $M$ such that $A$ is a complement of $X$ in $M$. Put $M=A \oplus B$. By the similar proof of Proposition 3.2, there exist an essential submodule $B^{\prime}$ of $B$ and homomorphisms $f: B^{\prime} \rightarrow A, g: B \rightarrow A$ such that

$$
\left\langle B^{\prime} \stackrel{f}{\rightarrow} A\right\rangle \subseteq_{e}\langle B \stackrel{g}{\rightarrow} A\rangle<_{\oplus} M \quad \text { and } \quad\left\langle B^{\prime} \stackrel{f}{\rightarrow} A\right\rangle \subseteq_{e} X .
$$

Thus $M$ is $\mathscr{G}$-extending.

Now we show that a $\mathscr{G}$-extending module with $\left(C_{3}\right)$ is just GQC with FIEP. 
THEOREM 3.4. Let $M$ be a module. Then

(1) If $M$ is $\mathscr{G}$-extending with $\left(C_{3}\right)$, then any direct summand of $M$ is $\mathscr{G}_{-}$ extending.

(2) $M$ is $\mathscr{G}$-extending with $\left(C_{3}\right)$ if and only if it is GQC with FIEP.

Proof. (1) Let $M=A \oplus B$ and let $X$ be a submodule of $A$. Since $M$ is $\mathscr{G}$-extending, there exist an essential submodule $X^{\prime}$ of $X$ and a direct summand $X^{*}$ of $M$ such that $X^{\prime} \subseteq_{e} X^{*}$. As $X^{*} \cap B=0, X^{*} \oplus B<_{\oplus} M$. So there exists a direct summand $K$ of $M$ such that $M=X^{*} \oplus K \oplus B$. By Lemma 1.3, there exists a homomorphism $\alpha: X^{*} \oplus K \rightarrow B$ such that $A=\left\langle X^{*} \stackrel{\left.\alpha\right|_{X^{*}}}{\longrightarrow} B\right\rangle \oplus\left\langle K \stackrel{\left.\alpha\right|_{K}}{\longrightarrow} B\right\rangle$. By $X^{\prime} \subseteq A \cap X^{*} \subseteq \operatorname{ker} \alpha$ and $X^{\prime} \subseteq_{e} X^{*}$, we see

$$
X^{\prime} \subseteq_{e}\left\langle X^{*} \stackrel{\left.\alpha\right|_{X^{*}}}{\longrightarrow} B\right\rangle .
$$

Thus $A$ is $\mathscr{G}$-extending.

(2) $(\Rightarrow)$ From [8, Proposition 16] (cf. [4, Theorem 2.15]), we may show that any decomposition $M=M_{1} \oplus M_{2}$ is exchangeable. Let $M=M_{1} \oplus M_{2}$ and let $X$ be a direct summand of $M$. By (1), $M_{i}$ is $\mathscr{G}$-extending and hence there exist an essential submodule $X_{i}^{\prime}$ of $M_{i} \cap X$ and a decomposition $M_{i}=A_{i} \oplus B_{i}$ such that $X_{i}^{\prime} \subseteq_{e} A_{i}(i=1,2)$. By Lemma 1.1, $X \supseteq_{e} X_{1}^{\prime} \oplus X_{2}^{\prime} \oplus\left(B_{1} \oplus B_{2}\right) \cap X$. As $B_{1} \oplus B_{2}$ is $\mathscr{G}$-extending, there exist an essential submodule $Y$ of $\left(B_{1} \oplus B_{2}\right) \cap X$ and a direct summand $T$ of $B_{1} \oplus B_{2}$ with $Y \subseteq_{e} T$. By $B_{1} \cap X=0$, we see $B_{1} \cap T=0$. Thus $B_{1} \oplus T$ is a direct summand of $B_{1} \oplus B_{2}$. Put $B_{1} \oplus B_{2}=L \oplus T \oplus B_{1}$. By Lemma 1.3, there exists a homomorphism $\alpha: L \oplus T \rightarrow B_{1}$ such that $B_{2}=$ $\left\langle L \stackrel{\left.\alpha\right|_{L}}{\rightarrow} B_{1}\right\rangle \oplus\left\langle T \stackrel{\left.\alpha\right|_{T}}{\rightarrow} B_{1}\right\rangle$. Put $\quad B_{2}^{\prime}=\left\langle L \stackrel{\left.\alpha\right|_{L}}{\rightarrow} B_{1}\right\rangle$. Then $\quad B_{1} \oplus B_{2}=T \oplus B_{1} \oplus B_{2}^{\prime}$. Thus we see

$$
X \supseteq_{e} X_{1}^{\prime} \oplus X_{2}^{\prime} \oplus Y \quad \text { and } \quad A_{1} \oplus A_{2} \oplus T \supseteq_{e} X_{1}^{\prime} \oplus X_{2}^{\prime} \oplus Y .
$$

So we see $\left(B_{1} \oplus B_{2}^{\prime}\right) \cap X=0$. By $\left(C_{3}\right), X \oplus B_{1} \oplus B_{2}^{\prime}$ is a direct summand of $M$. As $X \oplus B_{1} \oplus B_{2}^{\prime} \subseteq_{e} M$, we obtain $M=X \oplus B_{1} \oplus B_{2}^{\prime}$.

Therefore $M$ satisfies FIEP.

$(\Leftarrow)$ Let $A$ and $B$ be direct summands of $M$ with $A \cap B=0$. As $M$ is GQC, there exist essential submodules $A^{\prime} \subseteq_{e} A$ and $B^{\prime} \subseteq_{e} B$ and a decomposition $M=$ $M_{1} \oplus M_{2}$ such that $A^{\prime} \subseteq_{e} M_{1}$ and $B^{\prime} \subseteq M_{2}$. As $M_{2}$ is $\mathscr{G}$-extending, we may assume that $M=M_{1} \oplus M_{2} \oplus M_{3}$ with $A^{\prime} \subseteq_{e} M_{1}$ and $B^{\prime} \subseteq_{e} M_{2}$. As $M$ satisfies FIEP, there exists $M_{i}^{\prime} \subseteq M_{i}(i=1,2,3)$ with $M=A \oplus M_{1}^{\prime} \oplus M_{2}^{\prime} \oplus M_{3}^{\prime}$. By $M_{1} \supseteq_{e} A^{\prime} \subseteq_{e} A, M=A \oplus M_{2}^{\prime} \oplus M_{3}^{\prime}$. By $M_{3} \cap\left(A \oplus M_{2}^{\prime}\right)=0, M=A \oplus M_{2}^{\prime} \oplus M_{3}$. As $M$ satisfies FIEP, there exist $\bar{A} \subseteq A, \overline{M_{2}^{\prime}} \subseteq M_{2}^{\prime}$ and $\overline{M_{3}} \subseteq M_{3}$ with $M=$ 
$B \oplus \bar{A} \oplus \overline{M_{2}^{\prime}} \oplus \overline{M_{3}}$. By $M_{2} \supseteq_{e} B^{\prime} \subseteq_{e} B, M=B \oplus \bar{A} \oplus \overline{M_{3}}$. As $A \cap\left(B \oplus \overline{M_{3}}\right)=0$, we see $\bar{A}=A$. Thus $A \oplus B$ is a direct summand of $M$.

Finally, we touch on the relations of modules which are generalizations of quasi-continuous modules.

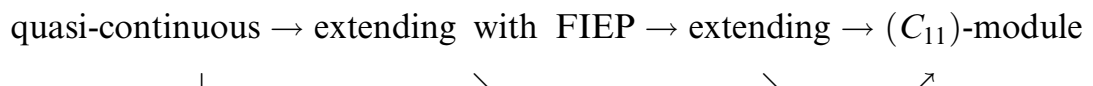

$\mathscr{G}$-extending with $\left(C_{3}\right) \rightarrow \mathscr{G}$-extending with FIEP $\rightarrow \mathscr{G}$-extending $\uparrow$

GQC-module with FIEP $\rightarrow$ GQC-module

\section{Acknowledgements}

The author is grateful to the referee for valuable comments and suggestions which improve the results and presentation of this article.

\section{References}

[ 1 ] Akalan, E., Birkenmeier, G. F. and Tercan, A., Goldie Extending Modules, Comm. Algebra 37 (2009), 663-683.

[2] Baba, Y. and Oshiro, K., Classical Artinian Rings and Related Topics, World Scientific Publishing Co. Pte. Ltd., 2009.

[ 3 ] Dung, N. V., Huynh, D. V., Smith, P. F. and Wisbauer, R., Extending Modules, Pitman Research Notes in Mathematics Series, Vol. 313, Longman, Harlow/New York, 1994.

[ 4 ] Hanada, K., Kuratomi, Y. and Oshiro, K., On direct sums of extending modules and internal exchange property, J. Algebra 250 (2002), 115-133.

[5] Keskin, Tütüncü D. and Kuratomi, Y., On mono-injective modules and mono-ojective modules, Math. J. Okayama Univ. 55 (2013), 117-129.

[6] Kuratomi, Y., On $\mathscr{G}$-extending modules with finite internal exchange property, submitted.

[ 7 ] Mohamed, S. H. and Müller, B. J., Continuous and Discrete Modules, London Math. Soc. LNS 147 Cambridge Univ. Press, Cambridge, 1999.

[ 8 ] Mohamed, S. H. and Müller, B. J., Ojective modules, Comm. Algebra 30 (2002), 18171827.

[9] Mohamed, S. H. and Müller, B. J., Co-ojective modules, J. Egptian Math. Soc. 12 (2004), 83-96.

[10] Oshiro, K., Continuous modules and quasi-continuous modules, Osaka J. Math. 20 (1983), 681-694.

[11] Oshiro, K. and Rizvi, S. T., The exchange property of quasi-continuous modules with the finite exchange property, Osaka J. Math. 33 (1996), 217-234.

[12] Smith, P. F. and Tercan, A., Generalizations of CS-modules, Comm. Algebra 21 (1993), 1809-1847.

[13] Smith, P. F. and Tercan, A., Direct summand of modules which satisfy $\left(C_{11}\right)$, Algebra Coll. 11 (2004), 231-237. 
[14] Wilson, G. V., Modules with summand intersection property, Comm. Algebra 14 (1986), 21-38.

[15] Wisbauer, R. Foundations of Module and Ring Theory, Gordon and Breach, Reading, 1991.

Department of Mathematics, Faculty of Science Yamaguchi University

1677-1 Yoshida, Yamaguchi, 753-8512, Japan

E-mail: kuratomi@yamaguchi-u.ac.jp 